\title{
PREVALENCE OF VIRULENCE FACTORS AMONG ENTEROCOCCAL ISOLATES IN A TERTIARY CARE HOSPITAL
}

Kadevari Swarnalathaํ, Manisha Singh², Sreekanth Basireddy³, Gundela Swarnalatha ${ }^{4}$

${ }^{1}$ Associate Professor, Department of Microbiology, Kurnool Medical College, Kurnool.

${ }^{2}$ Professor, Department of Microbiology, SVS Medical College, Mahbubnagar.

${ }_{3}^{3}$ Assistant Professor, Department of Microbiology, Kurnool Medical College, Kurnool.

4 Professor \& HOD, Department of Microbiology, Kurnool Medical College, Kurnool.

\section{ABSTRACT}

\section{BACKGROUND}

Enterococci are a notoriously resistant pathogen capable of producing an array of virulence factors. Biofilm production, gelatinase and haemolysin are the three potential virulence factors of Enterococci. Gelatinase and haemolysin producing strains of Enterococcus have been shown to cause severe infections in animal models. Biofilm production is associated with the persistence of Enterococcus in the medical indwelling devices and is extremely difficult to treat them.

\section{AIMS}

To know the prevalence of gelatinase, haemolysin and biofilm formation among clinical isolates of enterococci.

\section{MATERIALS AND METHODS}

A total of 76 enterococcal isolates obtained from various clinical samples during the period from July 2015 to 0 ctober 2015 were included in the study. All the isolates were speciated and tested for the presence of virulence factors which include gelatinase, haemolysin and biofilm production.

\section{RESULTS}

Out of 76 enterococcal isolates, E. faecalis were 31 (41\%) and E. faecium were 45 (59\%). Haemolysis was observed in 26 (34.2\%) isolates. Gelatinase was observed in 14 (18.4\%) isolates. Biofilm formation was observed in 28 (36.8\%) isolates. Biofilm production was more predominant in E. faecium isolates, whereas gelatinase production was more common in E. faecalis.

\section{CONCLUSION}

The study concludes that virulence determinants have been widely prevalent in enterococci isolates from clinical specimens. Prompt evaluation for identification of various virulence factors should be done, which in turn will help in the appropriate management of the patients.

\section{KEYWORDS}

Enterococcus, Gelatinase, Haemolysin, Biofilm Production, Virulence Factors.

HOW TO CITE THIS ARTICLE: Swarnalatha K, Singh M, Basireddy S, et al. Prevalence of virulence factors among enterococcal isolates in a tertiary care hospital. J. Evolution Med. Dent. Sci. 2016;5(51):3318-3322, DOI: 10.14260/jemds/2016/768

\section{INTRODUCTION}

Enterococci are increasingly being recognized as nosocomial pathogens and are associated with various infections. They have been isolated from postoperative wound infections, blood stream and urinary tract infections, intra-abdominal infections, pelvic infections and endocarditis. ${ }^{1}$ Though there are many different enterococci species identified in association with human disease, the majority of human enterococcal infections are due to the species Enterococcus faecalis and Enterococcus faecium.

The ability of Enterococcus isolates to cause serious infections is due to its intrinsic resistance of the bacterium to various components, which allows the organism to persist in the hospital environment and survive many host defences.

Financial or Other, Competing Interest: None.

Submission 05-05-2016, Peer Review 03-06-2016,

Acceptance 09-06-2016, Published 27-06-2016.

Corresponding Author:

Dr. Kadevari Swarnalatha,

Associate Professor,

Department of Microbiology,

Kurnool Medical College, Kurnool.

E-mail: swarnalatha@gmail.com

DOI: $10.14260 /$ jemds $/ 2016 / 768$
Above this it has the ability to acquire a variety of virulence traits by horizontal transfer from other organisms. ${ }^{2,3,4}$ A number of different virulence factors have been identified by many researchers, the most important among them being haemolysin, gelatinase, enterococcal surface protein (Esp), Aggregation Substance (AS), MSCRAMMAce (Microbial Surface Component Recognizing Adhesive Matrix Molecule Adhesion of Collagen from Enterococci), serine protease, capsule, cell wall polysaccharide and superoxide. ${ }^{5-11}$ A detailed study of the virulence factors can lead to a better understanding of the pathogenesis of enterococcal infections. Such virulence factors may play an important role in enhancing the pathogenicity and are expected to be associated with infections with a higher degree of severity as well as with nosocomial or hospital acquired infections.

Adherence to body surfaces is considered a major factor responsible for the pathogenicity of the clinical isolates of Enterococcus. Strains causing infection are having a greater capacity to adhere to surfaces than commensal strains. Biofilm formation plays a major role in nosocomial infections like catheter-associated UTIs, blood stream infections and endodontic infections. 
Haemolysin is a cytolytic protein capable of lysing human, horse and rabbit erythrocytes. Haemolysin-producing strains of E. faecalis have been shown to be virulent in animal models and human infections. ${ }^{22,13,14}$ and to be associated with increased severity of infection. 15 Gelatinase is a protease produced by E. faecalis that is capable of hydrolysing gelatin, collagen, casein, haemoglobin, and other peptides. ${ }^{16}$ Gelatinase-producing strains of E. faecalis have been shown to contribute to the virulence of endocarditis in an animal model. ${ }^{17}$

Out of an array of virulence factors, haemolysis, gelatinase and biofilm production are the most important ones and easily detectable in a routine diagnostic laboratory because of which these properties have been studied by many researchers. When associated with one or other of these virulence determinants, severity of the infections may be high and sometimes they may become refractory to the routine treatment, which needs aggressive treatment.

Treatment of Enterococcal infections is often complicated by antibiotic resistance. Apart from high level of resistance that is intrinsic to the species. ${ }^{18}$ acquired genes confer resistance to many antibiotics like chloramphenicol, clindamycin, erythromycin, tetracycline, high-level aminoglycosides and vancomycin rendering these drugs ineffective. 19

Only limited studies are available in the India with regards to the virulence factors detection. Our study aims to evaluate the prevalence of virulence determinants like biofilm formation, haemolysin production and gelatinase production in Enterococcus species isolated from clinical specimens.

\section{MATERIALS AND METHODS}

A total of 76 enterococcal isolates obtained from various clinical samples during the period from July 2015 to October 2015 were included in the study. Speciation of these enterococcal isolates was done by standard biochemical tests.(20) Virulence properties like haemolysis, gelatinase and biofilm production were detected by using the methodologies previously described. $(21,22)$

\section{Haemolysin Production}

Haemolysis production was observed by inoculating Enterococci on Mueller Hinton agar supplemented with 5\% human blood. Though sheep blood is commonly used for observing the haemolysis, human blood is also equally good and frequently used in healthcare setups because of the ease of availability. The inoculated plates were incubated overnight at $37^{\circ} \mathrm{C}$ and evaluated after 24 hours. A clear zone of beta haemolysis around the colonies was considered as positive. All the beta haemolytic streptococci were differentiated from enterococci by further testing on bile esculin agar.

\section{Gelatinase Production}

Gelatinase production was detected by inoculating the Enterococci on freshly prepared peptone yeast extract agar containing gelatin plates, incubated at $37^{\circ} \mathrm{C}$ overnight and cooled to ambient temperature for 2 hours. A turbid halo around the colonies was considered positive for gelatinase production. No halo around the colonies was considered negative.

\section{Biofilm Formation}

Biofilm formation was detected by using Congo red agar method. The Congo red test is based on the ability of this dye to stain polysaccharides black. The medium is composed of brain heart infusion broth supplemented with 5\% Sucrose, agar and Congo red stain. Plates were inoculated and incubated aerobically for 48 hours at $37^{\circ} \mathrm{C}$. Positive result was indicated by black colonies with a dry crystalline consistency. Dark colonies without dry crystalline colony morphology indicted moderate biofilm production. Weak biofilm producers produced dark pink colonies. Non-slime producers form dry pinkish red colonies. The experiment was performed in triplicate and repeated thrice.

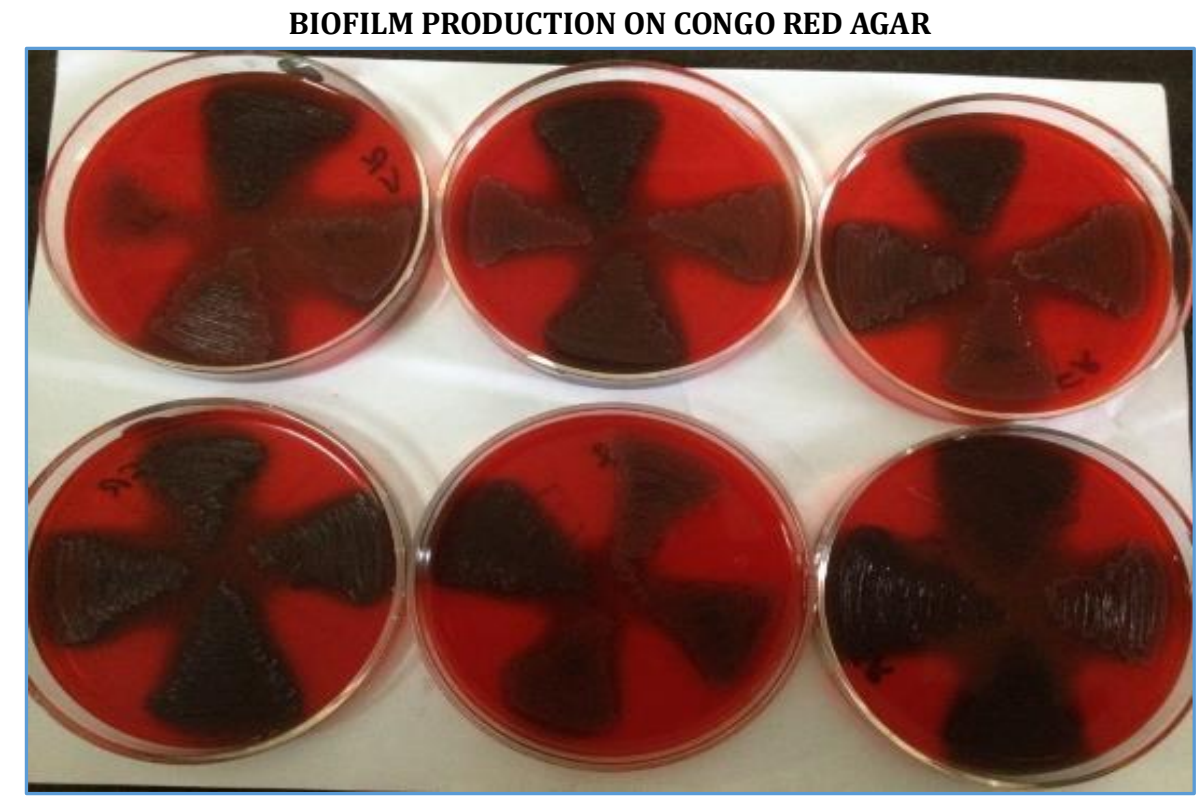

Fig. 1: Black Crystalline Colonies are the Biofilm Positive Strains and the Pinkish Red Colonies are Biofilm Negative 


\section{RESULTS}

Out of 76 enterococcal isolates, E. faecalis were 31 (41\%) and E. faecium were 45 (59\%), Haemolysis was observed in 26 (34.2\%) isolates. Gelatinase was observed in 14 (18.4\%) isolates. Biofilm formation was observed in 28 (36.8\%) isolates.

\begin{tabular}{|c|c|c|c|}
\hline $\begin{array}{c}\text { Virulence } \\
\text { Factors }\end{array}$ & $\begin{array}{c}\text { E. faecalis } \\
\mathbf{n = 3 1}\end{array}$ & $\begin{array}{c}\text { E. faecium } \\
\mathbf{n = 4 5}\end{array}$ & $\begin{array}{c}\text { Total } \\
\mathbf{( n = 7 6 )}\end{array}$ \\
\hline Haemolysin & 17 & 9 & $26(34 \%)$ \\
\hline Gelatinase & 14 & 0 & $14(18 \%)$ \\
\hline Biofilm & 5 & 23 & $28(39 \%)$ \\
\hline
\end{tabular}

E. faecalis: Among E. faecalis haemolysis was the most commonly observed virulence factor (54.8\%) followed by gelatinase production (45\%) and biofilm formation (16\%). All the three virulence properties were observed in only 4 $(12.9 \%)$ isolates. All these 4 isolates were from blood probably indicating its pathogenic property. One isolate was only biofilm and haemolysis positive but gelatinase negative, 10 isolates have shown both haemolysis and gelatinase property. Only haemolysis was seen in 2 isolates.

E. faecium: In E. faecium isolates biofilm formation was the most commonly observed virulence factor $(51 \%)$ followed by haemolysis production (20\%). None of the isolates have shown gelatinase production. All the 9 haemolytic strains showed biofilm production; 14 isolates showed only Biofilm production. Majority of the biofilm positive E. faecium were isolated from the urine samples, especially in catheterized patients.

\section{DISCUSSION}

Enterococci are one of the notoriously resistant pathogens, which are associated predominantly with nosocomial infections. They have the propensity to both acquire and spread genes responsible for drug resistance as well as they produce an array of other virulence factors, which are associated with disease causation. In the present study, we studied the two major species associated with most of the enterococcal infections namely, E. faecalis and E. faecium. Though many other species of enterococci are known to cause pathogenicity, E. faecalis and E. faecium are the commonly reported species from majority of the clinical specimens, out of which E. faecium is gaining importance and is the major isolated species in many centres now a days. Similarly, in our study E. faecium was the most commonly isolated species.

Though the prevalence of enterococcal infections has been increasing constantly, studies regarding the virulence factors detection in enterococci isolated from clinical as well as environmental samples have been scarcely done in India. ${ }^{23}$ However, even the limited studies document the widespread distribution of such traits. 24,25

Biofilm formation plays a major role in nosocomial infections, especially in catheter-associated UTIs and in device associated blood stream infections. They form biofilms on various indwelling devices like urinary catheters, prosthetic heart valves, artificial hip prostheses, etc. The biofilm formation is an important virulence factor, which is associated with refractoriness towards treatment. The ability of enterococci to form biofilms confers ecological advantage in certain situations. The clinical strains of E. faecalis isolated from infective endocarditis patients were significantly associated with the greater biofilm formation than nonendocarditis clinical isolates. ${ }^{26}$

A number of tests are available for the detection of biofilm production. These include the Tissue Culture Plate (TCP), Tube Method (TM), Congo Red Agar (CRA), Bioluminescent assay, piezoelectric sensors and light and fluorescent microscopic examination. Every method has got its own advantages and disadvantages and the methods described latter are beyond the scope of many laboratories and hospitals. Though tissue culture plate method is considered better methodology for the detection of biofilm formation, routine testing for the isolates by this method is not feasible in a resource poor settings where Congo red agar method is widely practiced. In our study biofilm formation was observed in $36.8 \%$ of the total isolates. Biofilm formation was predominantly seen in E. faecium isolates (51\%), whereas only $16 \%$ of the E. faecalis isolates have shown biofilm formation.

Gelatinase is an extracellular zinc-endopeptidase capable of hydrolysing gelatin and other peptides. Gelatinase production as a virulence factor in causing endocarditis has been studied using animal models. ${ }^{17}$ This enzyme helps enterococci by providing nutrition to the bacteria by degradation of host tissues. ${ }^{27}$ In our study, gelatinase production was seen in $18 \%$ of the isolates and all of them were E. faecalis isolates. None of the E. faecium isolates showed this property.

Haemolysin production is another virulent property of Enterococcus, which was shown to be associated with increased mortality in animal models of enterococcal endocarditis. In the present study, haemolysin production was seen in $34.2 \%$ of the total isolates. Haemolysin production was higher in E. faecalis (54.8\%) when compared to E. faecium (20\%).

Similar findings were observed in various studies conducted in India. In a study conducted by Giridhara Upadhyaya et al(28), haemolysin production was seen in only $16 \%$ of the isolates, whereas in Fernendes et al(24) study it was as high as $82 \%$. Our findings are intermediate to these two study findings. Similar to our findings gelatinase production was observed in 19\% of the isolates in Praharaj et al(25) study. Biofilm production was observed in $32.5 \%$ of the enterococcal isolates in Giridhara Upadhyaya et al(28) study, which is similar to our findings. In Banerjee et al(29) study, haemolysin, gelatinase and biofilm formation was observed in $23.22 \%, 8.3 \%$ and $25.16 \%$ of the E. faecalis isolates and $40 \%$ $9.6 \%$ and $27.09 \%$ among E. faecium isolates.

\begin{tabular}{|c|c|c|c|c|c|}
\hline $\begin{array}{c}\text { Virulence } \\
\text { Factors in Enterococci }\end{array}$ & $\begin{array}{c}\text { Giridhar } \\
\text { et al(28) }\end{array}$ & $\begin{array}{c}\text { Elsner HA } \\
\text { et al(30) }\end{array}$ & $\begin{array}{c}\text { Praharaj } \\
\text { et al(25) }\end{array}$ & $\begin{array}{c}\text { Fernandes } \\
\text { et al(24) }\end{array}$ & $\begin{array}{c}\text { Present } \\
\text { Study 2014 }\end{array}$ \\
\hline Haemolysis & $16 \%$ & $16 \%$ & $21 \%$ & $82 \%$ & $34.2 \%$ \\
\hline Gelatinase & $39 \%$ & $55 \%$ & $19 \%$ & $40.6 \%$ & $18.4 \%$ \\
\hline Biofilm & $32.5 \%$ & $63 \%$ & $53 \%$ & - & $36.8 \%$ \\
\hline
\end{tabular}


The present study identifies the prevalence and distribution of the three most common virulence factors which include haemolysis, gelatinase and biofilm production among the two most common enterococci species isolates from the clinical specimens. In view of the growing importance of Enterococcus species as nosocomial pathogens and the increasing prevalence of antibiotic resistance including glycopeptide resistance among enterococci, the identification of virulence factors which are associated with enterococcal invasiveness and disease severity will be an important subject of future investigations. Development of agents which can block enterococcal adherence or which can inhibit the action of other virulence factors may provide new therapeutic alternatives in the future.

\section{CONCLUSION}

Enterococci though once considered as harmless organisms, their importance is increasingly known now a days. With the expression of various virulence factors by the clinical isolates of enterococci, eradication of these infections have become much more difficult than ever. The present study conclude that virulence determinants have been widely prevalent in enterococci isolates from clinical specimens. Prompt evaluation for identification of various virulence factors should be done, which in turn will help in the appropriate management of the patients and decreases mortality and morbidity.

\section{REFERENCES}

1. Low DE, Keller N, Barth A, et al. Clinical prevalence, antimicrobial susceptibility, and geographic resistance patterns of enterococci: results from the SENTRY antimicrobial surveillance program, 1997-1999. Clin Infect Dis 2001;32(Suppl 2):S133-45.

2. Ike Y, Clewell DB. Genetic analysis of the pAD1 pheromone response in streptococcus faecalis, using transposon Tn 917 as an insertional mutagen. J Bacteriol 1984;158(3):777-83.

3. Shankar N, Baghdayan AS, Gilmore MS. Modulation of virulence within a pathogenicity island in vancomycinresistant enterococcus faecalis. Nature 2002;417(6890):746-50.

4. Dunny GM, Clewell DB. Transmissible toxin (haemolysin) plasmid in streptococcus faecalis and its mobilization of a noninfectious drug resistance plasmid. J Bacteriol 1975;124(2):784-90.

5. Hancock LE, Gilmore MS. Pathogenicity of Enterococci. In: Fischetti V, Novick R, Ferretti J, et al. (editors). Gram positive pathogens. Washington DC: Am Soc Microbiol 2000:251-8.

6. Garsin DA, Sifri CD, Mylonakis E, et al. A simple model host for identifying gram positive virulence factors. Proc Natl Acad Sci U S A 2001;98(19):10892-7.

7. Jett BD, Huycke MM, Gilmore MS. Virulence of enterococci. Clin Microbiol Rev 1994;7(4):462-78.

8. Nallapareddy SR, Qin X, Weinstock GM, et al. Enterococcus faecalis adhesion, ace mediates attachment to extracellular matrix proteins collagen type IV and laminin as well as collagen type I. Infect Immun 2000;68(9):5218-24.

9. Sartingen S, Rozdzinski E, Muscholl-Silberhorn A. Aggregation substances increases adherence and internalization but not translocation of enterococcus faecalis through different intestinal epithelial cells invitro. Infect Immun 2000;68(10):6044-7.
10. Hass W, Shepard BD, Gilmore MS. Two-component regulator of enterococcus faecalis cytolysin responds to quorum-sensing autoinduction. Nature 2002;415(6867):84-7.

11. Huycke MM, Abrams V, Moore DR. Enterococcus faecalis produces extracellular superoxide and hydrogen peroxide that damages colonic epithelial cell DNA. Carcinogenesis 2002;23(3):529-6.

12. Ike $Y$, Hashimoto $H$, Clewell DB. Haemolysin of streptococcus faecalis subspecied zymogenes contributes to virulence in mice. Infect Immun 1984;45(2):528-30.

13. Chow JW, Thal LA, Perri MB, et al. Plasmid-associated haemolysin and aggregation substance production contribute to virulence in experimental enterococcal endocarditis. Antimicrob Agents Chemother 1993;37(11):2474-7.

14. Ike $Y$, Hashimoto $H$, Clewell DB. High incidence of haemolysin production by enterococcus (Streptococcus) faecalis strains associated with human parenteral infections. J Clin Microbiol 1987;25(8):1524-8.

15. Johnson AP. The pathogenicity of enterococci. J Antimicrob Chemother 1994;33(6):1083-9.

16. Kreft B, Marre R, Schramm U, et al. Aggregation substance of enterococcus faecalis mediates adhesion to cultured renal tubular cells. Infect Immun 1992;60(1):25-30.

17. Gutschik E, Moller S, Christensen N. Experimental endocarditis in rabbits. 3. Significance of the proteolytic capacity of the infecting strains of streptococcus faecalis. Acta Pathol Microbiol Scand 1979;87(6):353-62.

18. Shepard BD, Gilmore MS. Antibiotic-resistant enterococci: the mechanisms and dynamics of drug introduction and resistance. Microbes Infect 2002;4(2):215-24.

19. Murray BE. The life and times of the enterococcus. Clin Microbiol Rev 1990;3(1):46-65.

20. Ross PW. Streptococcus and enterococcus. In: Collee JG, Fraser AG, Marmion BP, et al. (editors). Practical medical microbiology. New Delhi: Curchill Livingstone 2006: 263-73.

21. Vergis EN, Shankar N, Chow JW, et al. Association between the presence of enterococcal virulence factors gelatinase, haemolysin and enterococcal surface protein and mortality among patients with bacteraemia due to enterococcus faecalis. Clin Infect Dis 2002;35(5):570-5.

22. Freeman DJ, Falkiner FR. New method for detecting slime production by coagulase negative staphylococci. J Clin Pathol 1989;42(8):872-4.

23. Sood S, Malhotra M, Das BK, et al. Enterococcal infections \& antimicrobial resistance. Indian Journal of Medical Research 2008;128(2):111-21.

24. Fernandes SC, Dhanashree B. Drug resistance and virulence determinants in clinical isolates of enterococcus species. The Indian Journal of Medical Research 2013;137(5):981-5.

25. Ira P, Sujatha S, Chandra PS. Virulence factors in clinical and commensal isolates of enterococcus species. Indian Journal of Pathology and Microbiology 2013;56(1): 24-30. 
26. Christensen GD, Simpson WA, Bisno AL, et al. Adherence of slime-producing strains of staphylococcus epidermidis to smooth surfaces. Infect Immun 1982;37(1):318-26.

27. Fisher K, Phillips C. The ecology, epidemiology and virulence of enterococcus. Microbiology 2009;155(Pt 6):1749-57.

28. Girdhara PM, Umapathy BL, Ravikumar KL. Comparative study for the presence of enterococcal virulence factors gelatinase, haemolysin and biofilm among clinical and commensal isolates. J Lab Physicians 2010;2(2):100-4.
29. Banerjee T, Anupurba S. Prevalence of virulence factors and drug resistance in clinical isolates of enterococci: a study from north India. Journal of Pathogens Article ID 692612, 2015;2015:7 pages.

30. Elsner HA, Sobottka I, Mack D, et al. Virulence factors of enterococcus faecalis and enterococcus faecium blood culture isolates. Eur J Clin Microbiol Infect Dis 2000;19(1):39-42. 